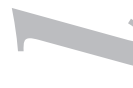

\title{
Enfermedad de Wilson. Una enfermedad rara pero presente
}

La enfermedad de Wilson (EW) o degeneración hepatolenticular, tal y como fue denominada por Kinnier Wilson en 1912 (1), es un trastorno del metabolismo del cobre que se hereda de forma autosómica recesiva (2). Su incidencia a nivel global es de 1 de cada 30.000 habitantes, aunque esta tiene una gran variabilidad geográfica. La EW se debe a mutaciones en el gen de una ATPasa tipo P (ATP7B) implicada en el transporte del cobre al canalículo biliar y al aparato Trans-Golgi.

El cobre procedente de la dieta, una vez captado por los enterocitos duodenales, puede quedar unido a proteínas intracelulares (metalotioneínas) o bien puede ser exportado al exterior a través de una proteína transportadora localizada en la membrana basolateral de esas células denominada ATP7A o ATPasa de Menkes (3). El gen que codifica esta proteína se expresa en todos los tejidos a excepción del hígado. Dicho gen se encuentra mutado en la enfermedad de Menkes, ligada al cromosoma X y caracterizada por un déficit de cobre consecuencia de una malabsorción de este metal. Una vez en la sangre, el cobre procedente del intestino se une a la albúmina o a la histidina, mientras que el de origen hepático se encuentra formando parte de la holoceruloplasmina. El cobre unido a estas moléculas, una vez reducido a su forma cuprosa, se une al transportador hCtrl de los hepatocitos y penetra en las células (4). Por su alto poder oxidante, el cobre debe unirse a diversas proteínas celulares, fundamentalmente a las metalotioneínas, que neutralizan ese poder (5). Otras proteínas de pequeño tamaño denominadas metalochaperonas hacen de transportadores de cobre dentro de las células (6). Entre estas, la ATOX1 transporta el cobre hasta la ATP7B. Esta enzima tiene seis zonas ricas en cisteína y metionina a las que se une este metal (7). La localización de la ATP7B dentro de los hepatocitos no es única y puede oscilar entre el aparato Trans-Golgi y el capilar biliar, dependiendo ello de la concentración de cobre en las células. Cada uno de estos emplazamientos está ligado a una función diferente. La primera se relaciona con la formación de homoceruloplasmina y el paso del cobre a la sangre; la segunda con la excreción del cobre con la bilis (8). En condiciones normales, cuando la concentración de cobre es baja, la ATP7B se localiza en la zona Trans del aparato de Golgi y permite la unión del cobre a la apoceruloplasmina para formar holoceruloplasmina antes de su paso a la sangre. Cuando los niveles intracelulares de cobre son altos, la ATP7B se desplaza al polo biliar de los hepatocitos, lo que facilita la excreción biliar del cobre (9). No se conoce con detalle cuál es el mecanismo molecular por el que la ATP7B consigue la excreción del cobre por el polo biliar, pero se supone que la proteína Murr1 o COMMD1 juega un importante papel (10).

La ATP7B es codificada por el gen $A T P 7 B$ localizado en el brazo largo del cromosoma 13 (13q14.3) (11-14). En la EW se han descrito unas 300 mutaciones del este gen, pero en la mayoría de los casos la enfermedad es ocasionada por la asocia- 
ción de dos mutaciones heterozigotas. Cuando esto ocurre, el cobre no puede ser vertido a la bilis y, en consecuencia, se acumula en los hepatocitos. Asimismo, el cobre tampoco puede pasar al Trans-Golgi, no se puede unir a la apoceruloplasmina y no se forma holoceruloplasmina. Cuando la apoceruloplasmina pasa al plasma, es eliminada rápidamente, ya que su vida media es más corta que la de la holoceruloplasmina. La escasa cantidad de cobre que pasa a la sangre lo hace en su forma libre, no unida a la ceruloplasmina, por lo que se deposita fácilmente en distintos tejidos, principalmente en el sistema nervioso, el túbulo renal o la córnea, dando lugar a algunas de las manifestaciones que caracterizan a esta enfermedad.

La mutación más frecuente en pacientes europeos con EW es la sustitución de una histidina por glutamato en la posición 1069 (H1069Q), que se encuentra en el 40 al $60 \%$ de todos los casos (15). Esta mutación influye en la utilización de ATP y en consecuencia en la adquisición de la energía que requiere el transporte del cobre (16). En España, se ha encontrado que la mutación más frecuente es la M645R, presente en el 55\% de los pacientes. La frecuencia de las restantes mutaciones es mucho más baja $(17,18)$, si bien en las Islas Canarias se ha descrito una alta prevalencia de la mutación L708P (19). Sería interesante conocer las mutaciones existentes en la población estudiada por el grupo de Rodrigo Agudo (20).

No se conocen aún bien cuáles son los mecanismos por los que el cobre ejerce su toxicidad celular (21). Sabiendo que el cobre es un agente prooxidante, se ha considerado que el estrés oxidativo podría jugar un papel importante. A través de la vía de Fenton y de otras reacciones redox, tanto el hierro $(22,23)$ como el cobre $(24,25)$ pueden provocar estrés oxidativo y la peroxidación lipídica, dando lugar a lesiones de membrana y disfunción de las organelas celulares (26). Es bien sabido que en esta enfermedad existen importantes anomalías morfológicas y funcionales de las mitocondrias (27). Este mecanismo podría justificar el empleo de antioxidantes en estos enfermos. Otro mecanismo recientemente sugerido por el que el cobre podría provocar la muerte de los hepatocitos y la anemia se basa en su capacidad para activar a la esfingomielinasa ácida y liberar ceramida. Tanto la deficiencia genética como la inhibición farmacológica de la esfingomielinasa ácida previenen la apoptosis hepatocitaria y el desarrollo de hepatopatía en modelos murinos de EW. La activación de la esfingomielinasa ácida conlleva la liberación de fosfatidilserina a la sangre. Los hematíes expuestos a esta última se destruyen rápidamente, lo que explicaría la anemia de la EW (28). Algunos estudios indican que, al menos en las fases iniciales de la EW, las lesiones pueden no estar relacionadas con el efecto directo del cobre sino con alteraciones en el metabolismo del colesterol y otras vías metabólicas específicas (29).

En el presente número de esta revista, Rodrigo Agudo y cols. recogen la experiencia reunida en un solo centro sobre la EW (20). Este estudio realizado en la región de Murcia se suma a otros publicados previamente y realizados en otras regiones de España $(30,31)$. En su estudio, los autores refieren las características demográficas de sus enfermos, las formas de presentación de la enfermedad, las alteraciones analíticas, el tratamiento establecido y la evolución a lo largo de unos diez años. La enfermedad rara vez se diagnostica antes de los 3 años de edad y la mayoría de los casos se detecta durante la adolescencia como enfermedad hepática. Como refieren los autores de este estudio, la mayor parte de los casos descritos en la literatura se reconocen a partir de una elevación asintomática de las transaminasas. En algunos casos, la enfermedad se diagnostica en edades más avanzadas, por encima de los 50 años, coincidiendo con una enfermedad también más evolucionada y teniendo, por tanto, peor pronóstico. Aunque ninguno de los pacientes incluidos en 
la serie ahora publicada lo presentaba, diversos autores han comunicado la posibilidad de que la enfermedad hepática se manifieste como una hepatitis no infecciosa, que puede simular una hepatitis autoinmune (32). Como ocurre en estas hepatitis, los pacientes refieren astenia, artropatía, erupción cutánea, hipergammaglobulinemia e incluso la positividad de los anticuerpos antinucleares (ANA) y antimúsculo liso (SMA). El correcto reconocimiento de la etiología de estos casos es fundamental para indicar el tratamiento más adecuado (33). La presentación de la enfermedad en forma de fallo hepático agudo se describe en el 5-15\% de los trasplantes hepáticos infantiles, en ocasiones como primera manifestación de la EW (34). Estos casos suele cursar con anemia hemolítica intravascular y prueba de Coombs negativa (35). En este contexto, es característico hallar niveles extremadamente bajos de fosfatasa alcalina y comparativamente reducidos de transaminasas y de ácido úrico en sangre. Por ello, la presencia de un cociente de fosfatasa alcalina (en IU/l) frente a bilirrubina $($ en $\mathrm{mg} / \mathrm{dl})<2$ es típica de la insuficiencia hepática aguda por EW (36-38). No todos han confirmado la utilidad diagnóstica del bajo cociente fosfatasa alcalina/bilirrubina (39).

Como señalan Rodrigo Agudo y cols. en su estudio, la forma de presentación neuropsiquiátrica es más frecuente en los adultos, aunque puede detectarse alguna alteración de este tipo hasta en la mitad de los adolescentes (40). En su estudio, las manifestaciones neurológicas más frecuentes fueron los temblores, la disartria y la distonía, que las encontraron hasta en el 17\% de los pacientes. Estos resultados son coincidentes con lo referido en la literatura, ya que los temblores y la disartria son más frecuentes que la distonía. Dentro de las manifestaciones psiquiátricas, los cambios en la personalidad y la irritabilidad son las más frecuentes, aunque algunos pacientes se presentan con depresión o con un cuadro psicótico (41).

Otras manifestaciones menos comunes de la EW son la litiasis biliar de bilirrubinato, justificada por las crisis intermitentes de hemólisis y por la cirrosis hepática, cuando esta existe. Es bien conocido el anillo de Kayser y Fleischer, que en el estudio comentado estaba presente en el 35,3\% de los enfermos con manifestaciones exclusivamente hepáticas, pero en todos los que presentaban manifestaciones neurológicas o psiquiátricas, y las cataratas en girasol, determinadas por el depósito de cobre en el cristalino (42). A nivel endocrinológico se describe la ginecomastia en los varones, la amenorrea en las mujeres o hipoparatiroidismo (43). Aunque el embarazo es posible, este se ve dificultado y existe un riesgo aumentado de abortos y otras complicaciones (44). También se han descrito algunas manifestaciones cardiovasculares, entre las que figuran anomalías en el electrocardiograma (50\%), hipotensión ortostática $(20 \%)$ y la fibrilación ventricular $(45,46)$. Los pacientes también pueden sufrir urolitiasis o, con mayor frecuencia, alteraciones funcionales del túbulo renal proximal expresadas por un síndrome de Fanconi (47). Aunque clásicamente se ha creído que el riesgo de carcinoma hepatocelular es bajo en la EW, estudios recientes indican que este tumor es más frecuente de lo que se piensa $(48,49)$, por lo que los pacientes con cirrosis también deben ser sometidos a vigilancia para detectar precozmente el desarrollo de este tumor (50). También son varios los autores que refieren el desarrollo de colangiocarcinomas en estos enfermos $(49,51)$ y en ratones con silenciamiento del gen ATP7B (52).

Con respecto al diagnóstico de la enfermedad, se puede considerar que una ceruloplasmina menor a $5 \mathrm{mg} / \mathrm{dl}$ es prácticamente diagnóstica de EW en un paciente con alteraciones hepáticas o clínica neurológica típica. La presencia de una ceruloplasmina menor a $20 \mathrm{mg} / \mathrm{dl}$ aparece en el $95 \%$ de los pacientes y en el $20 \%$ de los heterocigotos asintomáticos, aunque no es suficiente para el diagnóstico (53). No obs- 
tante, puede ser normal en algunos casos por tratarse de un reactante de fase aguda (54) y puede estar descendida en la enfermedad de Menkes, en la aceruloplasminemia y en las hipoproteinemias (55). Si combinamos estos niveles de ceruloplasmina con el hallazgo del anillo de Kayser- Fleischer en el examen oftalmológico o con unos niveles altos de cobre en el tejido obtenido en la biopsia hepática ( $>250 \mu \mathrm{g} / \mathrm{g}$ de peso seco), la especificidad aumenta considerablemente. La medición del cobre en el tejido hepático es probablemente la prueba más específica para el diagnóstico de EW. No obstante, existen falsos negativos (intensa fibrosis y escasos hepatocitos, distribución irregular del cobre) y falsos positivos (colestasis) (56,57). Menos sensibles son las técnicas histoquímicas de detección de cobre en el tejido hepático, ya estas son positivas únicamente en las fases avanzadas de la enfermedad. En la EW el cobre urinario de 24 horas suele ser mayor de $40 \mu \mathrm{g}$ y muchas veces mayor de $100 \mu \mathrm{g}(58,59)$. Tras administrar D-penicilamina $500 \mathrm{mg}$ cada 12 horas por vía oral, se produce un aumento considerable de la cupruria, tomándose niveles superiores a $1.600 \mu \mathrm{g}$ como diagnósticos. Sin embargo, esta prueba ha sido validada sólo en la infancia, con el fin de diferenciar la EW de otras hepatopatías, por ejemplo, la hepatitis autoinmune (60); no obstante, algunos estudios sugieren que también puede ser útil en los adultos (61).

El anillo de Kayser-Fleischer es típico de la enfermedad aunque no patognomónico, ya que se puede encontrar también en enfermedades colestásicas crónicas. Este anillo se encuentra en el 50\% de los pacientes con manifestaciones hepáticas, como lo muestra el estudio de Rodríguez Agudo y cols., y en prácticamente todos los pacientes con manifestaciones neurológicas. Sólo en el $2 \%$ de estos últimos pacientes falta este hallazgo.

Puesto que ninguna prueba permite excluir o diagnosticar la EW con absoluta seguridad, se han intentado crear sistemas diagnósticos basados en puntuaciones que estarían especialmente indicados en los casos atípicos, de presentación tardía, en ausencia de sintomatología neuropsiquiátrica típica o de bioquímica característica. Uno de ellos es el sistema aceptado en el VIII Congreso Internacional de la Enfermedad de Wilson y Enfermedad de Menkes, en Leipzig. Este sistema, clasifica la enfermedad según su forma de presentación en: $H 1$ cuando es hepática aguda, $H 2$ cuando es hepática crónica, $N 1$ cuando debuta con sintomatología neuropsiquiátrica y hepática, $N 2$ cuando tiene síntomas neurológicos pero la afectación hepática es asintomática, $N X$ cuando no hay alteraciones hepáticas de ningún tipo y $O$ cuando debuta con otra sintomatología $(57,62,63)$.

Algunos autores defienden la importancia del diagnóstico genético a pesar de que las restantes pruebas sean negativas, sobre todo en niños con enfermedad hepática no filiada o con histología compatible con enfermedad grasa del hígado no alcohólica (64).

A pesar de que la base molecular de la EW es siempre la misma, llama la atención la marcada heterogeneidad de las manifestaciones clínicas de esta enfermedad. Inicialmente se atribuyó a la gran cantidad de mutaciones descritas en este gen. Sin embargo, es muy probable que también influyan otros factores. En realidad, es extremadamente complejo demostrar que existe alguna correlación entre genotipo y el fenotipo de la EW, pues la mayoría de los pacientes son heterocigotos compuestos. En un estudio reciente, hasta el 77\% de los pacientes con la mutación H1069Q la tenía con carácter heterocigoto (65). En un metaanálisis se ha encontrado que los pacientes homocigotos para esta mutación tienen una presentación neurológica tardía (66). Sin embargo, existen familias, incluso gemelos idénticos con la misma mutación, en quienes las manifestaciones clínicas son distintas. Por último, el 15\% de los 
pacientes diagnosticados de la forma clásica de EW no tiene ninguna mutación conocida en el gen ATP7B $(67,68)$.

Los familiares de primer grado de casos probados deben ser sometidos a estudio para reconocer tempranamente la presencia de la enfermedad. Estos estudios deben incluir la determinación del: perfil hepático, fundamentalmente de las transaminasas, del cobre en plasma y de la ceruloplasmina sérica, la cupruria de 24 horas y la búsqueda del anillo de Kayser-Fleischer mediante lámpara de hendidura. En caso de hallar alguna anomalía en las pruebas mencionadas previamente, se procederá a la realización de biopsia hepática. El análisis mutacional en el momento actual es difícil, ya que la mayoría de los pacientes son heterocigotos combinados y también debido a que no siempre las mutaciones reportadas producen proteínas no funcionantes. Este método de estudio de los familiares de pacientes con EW puede ser útil en aquellos casos en los que ya se conoce el genotipo del caso índice o en determinadas áreas geográficas en las que sabemos que el espectro mutacional es escaso, como en Islandia, las Islas Canarias (19) o Cerdeña. Únicamente los descendentes que sean homocigotos o heterocigotos compuestos para mutaciones conocidas deben recibir tratamiento preventivo con quelantes. Si son heterocigotos simples, esta profilaxis no es necesaria.

En relación con el tratamiento, la terapia más efectiva sigue siendo la utilización de quelantes de cobre fundamentalmente la D-penicilamina. La enfermedad sigue un curso fatal sin tratamiento médico o trasplante. En el modelo animal de la EW (rata Long-Evans Cinnamon) la administración de D-penicilamina produce la disolución de los gránulos de cobre que se encuentran en los lisosomas y evita el fallo hepático agudo. Otras alternativas son la trientina y el zinc. La trientina tiene un mecanismo similar pero una estructura molecular completamente distinta a la de la D-penicilamina. El mecanismo de acción del zinc es distinto, ya que actúa bloqueando la absorción intestinal de cobre (69). El zinc aumenta las metalotioneínas en los enterocitos, el cobre se une a ellas y se retiene en las células intestinales hasta que estas se descaman en la luz del intestino. De esta forma, el zinc provoca un balance negativo de cobre que conduce a una depleción del cobre del organismo. El aumento de metaloproteínas ocurre también en los hepatocitos, lo cual contribuye a proteger a estas células frente al efecto tóxico del cobre. Un problema importante es la adherencia terapéutica, ya que se debe administrar tres veces al día, separado de las comidas. Si el tratamiento es eficaz, debe descender el cobre libre en plasma y la cupruria, desaparecer el anillo de Kayser-Fleischer y normalizar la analítica hepática. Este tratamiento, de efectos más lentos, se recomienda en los sujetos asintomáti$\cos$, en familiares presintomáticos o una vez que se ha conseguido la depleción de cobre mediante tratamiento quelante (70). El tratamiento con agentes quelantes, como la D-penicilamina o la trientina, induce la pérdida por orina de 3 a $8 \mu \mathrm{mol}$ de cobre al día. Durante el tratamiento con zinc, la cupruria suele ser menor a 1,2 $\mu \mathrm{mol}$ al día. Ninguno de estos tratamientos ha sido evaluado de forma comparativa (71). El tetratiomolibdato amónico se recomienda en las formas neurológicas, ya que el empeoramiento neurológico durante este tratamiento quelante es mucho más raro $(4 \%)$ que con la D-penicilamina $(50 \%)$ o la trientina $(71,72)$. En la mayoría de los estudios, el deterioro neurológico secundario al tratamiento con quelantes es un factor predictivo de mortalidad (72,73). Los antioxidantes (vitamina E) pueden emplearse como adyuvantes. Se recomienda también evitar la toma de alimentos ricos en cobre como el hígado, marisco, champiñones, chocolate o frutos secos.

El trasplante hepático está indicado en casos con enfermedad hepática muy avanzada, cuando no se observa respuesta al tratamiento y en los pacientes con he- 
patitis fulminante, incluida la que puede surgir como consecuencia del abandono de los quelantes (71). Se puede considerar el trasplante de donante vivo familiar, aun cuando posea como máximo una única mutación. Los resultados del trasplante son excelentes en los pacientes con manifestaciones predominantemente hepáticas con una supervivencia anual del $90 \%$, del $83 \%$ a los 3 años y del $70 \%$ a los 5 años (7476). Los pacientes con manifestaciones neurológicas pueden mejorar también tras el trasplante hepático, pero su supervivencia es mucho menor que cuando faltan estos síntomas. Por ello, muchos autores consideran que el trasplante hepático no debe realizarse en pacientes que presentan exclusivamente manifestaciones neurológicas o cuando estas son graves si se asocian con enfermedad hepática $(74,77,78)$. Además, en estos pacientes, al igual que en los que presentan manifestaciones renales, el empleo de inhibidores de la calcineurina puede seguirse de síntomas neurológicos, a veces graves (79). Los síntomas psiquiátricos no suelen desaparecer, por lo que se considera que estos síntomas, al igual que la sintomatología neurológica grave, pueden constituir una contraindicación para el trasplante (74). Afortunadamente, los pacientes analizados por el grupo de Rodrigo Agudo no presentaron problemas especiales tras el trasplante hepático y todos los enfermos evolucionaron favorablemente, a pesar de que el 37,9\% de ellos presentaba síntomas neurológicos o psiquiátricos. El trasplante de hepatocitos, que constituye una esperanza para el futuro, requiere también del empleo de inmunosupresores (80). Quizá el empleo de células madres o de células modificadas mediante terapia génica podría reducir los riesgos de la inmunosupresión.

Contamos con estudios prometedores de terapia génica realizados en un modelo animal de EW, utilizando ratas Long Evans, en el que se ha logrado la normalización de la excreción biliar de cobre y el aumento de la ceruloplasmina sérica mediante la introducción del gen ATP7B incorporado a un lentivirus recombinante. Para que la infección por este virus sea eficaz, es suficiente con que este gen se exprese en el 30 al $50 \%$ de los hepatocitos $(81,82)$. Uno de los principales problemas que plantea esta terapia es el carácter transitorio de la expresión génica. Ello obliga a la repetición de la infección con el portador viral. Por ello, se necesita conseguir vectores genéticos capaces de infectar hepatocitos no proliferativos y que expresen de forma prolongada la enzima en la región celular más adecuada para conseguir una función óptima.

P. Solís Muñoz y J. A. Solís Herruzo

Servicio de Medicina del Aparato Digestivo. Hospital Universitario "12 de Octubre”. Madrid

\section{Bibliografía}

1. Wilson SAK. Progressive lenticular degeneration: a familial nervous disease associated with cirrhosis of the liver. Brain 1912; 34: 20-509.

2. Scheinberg IH, Sternlieb I. Wilson's disease. Philadelphia: WB Saunders; 1984.

3. Yamaguchi Y, Heiny ME, Gitlin JD. Biochemical characterization and intracellular localization of Menkes disease protein. Proc Natl Acad Sci USA 1996; 93: 14030-5.

4. Guo Y, Smith K, Lee J, Petris MJ. Identification of methionine-rich clusters that regulate copper-stimulated endocytosis of the human Crt1 copper transporter. J Biol Chem 2004; 279: 17428-33. 
5. Harris ED. Cellular copper transport and metabolism. Annu Rev Nutr 2000; 20: 291-310.

6. Field LS, Luk E, Carlotta VC. Copper chaperones: personalescorts for metal ions. J Bioenerg Biomembr 2002; 34: 373-9.

7. Walter JM, Huster D, Ralle M, Morgan CT, Blackburn NJ, Lutsenko S. The N-terminal metal-binding site 2 of the Wilson's disease protein plays a key role in the transfer of copper from Atox1. J Biol Chem 2004; 279 : $15376-84$.

8. Lutsenko S, Barnes NL, Bartee MY, Dmitriev OY. Function and regulation of human copper-transporting ATPases. Physiol Rev 2007; 87: 1011-46.

9. Schaefer M, Roelofsen H, Wolters H, Hofmann WJ, Müller M, Kuipers F, et al. Localization of the Wilson's disease protein in human liver. Gastroenterology 1999; 117: 1380-5.

10. Tao TY, Liu F, Klomp L, Gitlin JD. The copper toxicosis gene product Murr1 directly interacts with the Wilson disease protein. J Biol Chem 2003; 278: 41593-6.

11. Frydman M, Bonne-Tamir B, Farrer LA, Conneally PM, Magazanik A, Ashbel S, et al. Assignment of the gene for Wilson disease to chromosome 13: linkage to the esterase D locus. Proc Natl Acad Sci USA 1985 82: 1819-21.

12. Bull PC, Thomas GR, Rommens JM, Forbes JR, Cox DW. The Wilson disease gene is a putative copper transporting P-type ATPase similar to the Menkes gene. Nat Genet 1993; 5: 327-37.

13. Tanzi RE, Petrukhin K, Chernov I, Pellequer JL, Wasco W, Ross B, et al. The Wilson disease gene is a copper transporting ATPase with homology to the Menkes disease gene. Nat Genet 1993; 5: 344-50.

14. Yamaguchi Y, Heiny ME, Gitlin JD. Isolation and characterization of a human liver cDNA as a candidate gene for Wilson disease. Biochem Biophys Res Commun 1993; 197: 271-7.

15. Ala A, Walter AP, Ashkan K, Dooley JS, Schilsky ML. Wilson's disease. Lancet 2007; 369: 397-408.

16. Tsivkovskii R, Efremov RG, Lutsenko S. The role of the invariant His-1069 in folding and function of the Wilson's disease protein, the human copper-transporting ATPase ATP7B. J Biol Chem 2003; 278: 13302-8.

17. Margarit E, Bach V, Gómez D, Bruguera M, Jara P, Queralt R, et al. Mutation analysis of Wilson disease in the Spanish population -identification of a prevalent substitution and eight novel mutations in the ATP7B gene. Clin Genet 2005; 68: 61-8

18. Ferenci P. Regional distribution of mutations of the ATP7B gene in patients with Wilson disease: impact on genetic testing. Hum Genet 2006; 120: 151-9.

19. García-Villarreal L, Daniels S, Shaw SH, Cotton D, Galván M, Gestes J, et al. High prevalence of the very rare Wilson disease gene mutation Leu708Pro in the Island of Gran Canaria (Canary Islands, Spain): a genetic and clinical study. Hepatology 2000; 32: 1329-36.

20. Rodrigo Agudo JL, Valdés Mas M, Vargas Acosta AM, Ortiz Sánchez ML, Gil del Castillo ML, Carballo Álvarez LF, et al. Presentación clínica, diagnóstico y evolución a largo plazo en 29 pacientes con enfermedad de Wilson. Rev Esp Enferm Dig 2008; 100: 456-61.

21. de Bie P, Muller P, Wijmenga C, Klomp LW. Molecular pathogenesis of Wilson and Menkes disease: correlation of mutations with molecular defects and disease phenotypes. J Med Genet 2007; 44: 673-88.

22. Masini A, Ceccarelli D, Giovannini F, Montosi G, Garuti C, Pietrangelo A. Iron-induced oxidant stress leads to irreversible mitochondrial dysfunctions and fibrosis in the liver of chronic iron-dosedgerbils. The effect of silybin. J Bioenerg Biomembr 2000; 32: 175-82.

23. Eaton JW, Qian MW. Molecular bases of cellular iron toxicity. Free Radic Biol Med 2002; 32: 833-40.

24. Gaetke LM, Chow CK. Copper toxicity, oxidative stress, and antioxidant nutrients. Toxicology 2003; 189: 147-63.

25. Krumschnabel G, Manzl C, Berger C, Hofer B. Oxidative stress, mitochondrial permeability transition, and cell death in $\mathrm{Cu}$-exposed trout hepatocytes. Toxicol Appl Pharmacol 2005; 209: 62-73.

26. Aaseth J, Flaten TP, Andersen O. Hereditary iron and copper deposition: diagnostics, pathogenesis and therapeutics. Scand J Gastroenterol 2007; 42: 673-81.

27. Gu M, Cooper JM, Butler P, Walker AP, Mistry PK, Dooley JS, et al. Oxidative phosphorilation defects in liver of patients with Wilson's disease. Lancet 2000; 356: 469-74.

28. Lang PA, Schenck M, Nicolay JP, Becker JU, Kempe DS, Lupescu A, et al. Liver cell death and anemia in Wilson disease involve acid sphingomyelinase and ceramide. Nature Medicine 2007; 13: 164-70.

29. Huster D, Lutsenko S. Wilson disease: not just a copper disorder. Analysis of a Wilson disease model demonstrates the link between copper and lipid metabolism. Mol Biosyst 2007; 3: 816-24.

30. Sempere E, Pérez-Aguilar F, Burguera JA, Berenguer J. Enfermedad de Wilson: dificultades en el diagnóstico y manejo terapéutico en nuestro país. Rev Clin Esp 1989; 185: 348-53.

31. Castellano G, Blasco A, Ballesta F, Colina F, Moreno D, Franch O, et al. Enfermedad de Wilson. Análisis retrospectivo de doce casos. Rev Clin Esp 1992; 190: 223-8.

32. Milkiewicz P, Saksena S, Hubscher SG, Elias E. Wilson's disease with superimposed autoimmune features: report of two cases and review. J Gastroenterol Hepatol 2000; 15: 570-4.

33. Santos RG, Alissa F, Reyes J, Teot L, Ameen N. Fulminant hepatic failure: Wilson's disease or autoimmune hepatitis? Implications for transplantation. Pediatr Transplant 2005; 9: 112-6.

34. Ostapowicz G, Fontana RJ, Schiodt FV, Davern TJ, Lalani E, Hynan LS, et al. U.S. Acute Liver Failure Study Group. Results of a prospective study of acute liver failure at 17 tertiary care centers in the United States. Ann Intern Med 2002; 137: 947-54.

35. Saito T. Presenting symptoms and natural history of Wilson disease. Eur J Pediatr 1987; 146: 261-5.

36. Tissieres P, Chevret L, Debray D, Devictor D. Fulminant Wilson's disease in children: appraisal of a critica diagnosis. Pediatr Crit Care Med 2003; 4: 338-43.

37. Sallie R, Katsiyiannakis L, Baldwin D, et al. Failure of simple biochemical indexes to reliably differentiate fulminant Wilson's disease from other causes of fulminant liver failure. Hepatology 1992; 16: 1206-11.

38. Berman DH, Leventhal RI, Gavaler JS, Cadoff EM, Van Thiel DH. Clinical differentiation of fulminant Wilsonian hepatitis from other causes of hepatic failure. Gastroenterology 1991; 100: 1129-34.

39. Eisenbach C, Sieg O, Stremmel W, Encke J, Merle U. Diagnostic criteria for acute liver failure due to Wilson disease. World J Gastroenterol 2007; 13: 1711-4 
40. Brewer GJ. Neurologically presenting Wilson's disease: epidemiology, pathophysiology and treatment. CNS Drugs 2005; 19: 185-92.

41. Rathbun JK. Neuropsychological aspects of Wilson's disease. Int J Neurosci 1996; 85: 221-9.

42. Cairns JE, Williams HP, Walshe JM. "Sunflower cataract" in Wilson's disease. BMJ 1969; 3: 95-6.

43. Carpenter TO, Carnes DL Jr. Anast CS. Hypoparathyroidism in Wilson's disease. Liver 1992; 12: 42-5.

44. Tarnacka B, Rodo M, Cichy S, Czlonkowska A. Procreation ability in Wilson's disease. Acta Neurol Scand 2000; 101: 395-8.

45. Kuan P. Cardiac Wilson's disease. Chest 1987; 91: 579-83.

46. Hlubocka Z, Maracek Z, Linhart A, et al. Cardiac involvement in Wilson disease. J Inher Metab Dis 2002; 25: 269-77.

47. Azizi E, Eshel G, Aladjem M. Hypercalciuria and nephrolithiasis as a presenting sign in Wilson disease. Eur J Pediatr 1989;148:548-9

48. Iwadate H, Ohira H, Suzuki T, et al. Hepatocellular carcinoma associated with Wilson's disease. Intern Med 2004; 43: 1042-5.

49. Walshe JM, Waldenstrom E, Sams V, Nordlinder H, Westermark K. Abdominal malignancies in patients with Wilson's disease. QJM 2003: 96: 657-62.

50. Kumagi T, Horiike N, Abe M, et al. Small hepatocellular carcinoma associated with Wilson's disease. Intern Med 2005: 44: 439-43.

51. Ponomarev AB, Kosminkova EN, Generalova S. Diffuse cholangiocarcinoma in the context of multilobular liver cirrhosis as a manifestation of Wilson-Konovalov disease. Arkh Patol 1994; 56: 74-7.

52. Huster D, Finegold MJ, Morgan CT, Burkhead JL, Nixon R, Vanderwerf SM, et al. Consequences of copper accumulation in the livers of the Atp7b-/- (Wilson disease gene) knockout mice. Am J Pathol 2006; 168: 42334.

53. Roberts EA, Schilisky ML. A practice guideline on Wilson disease. Hepatology 2003; 37: 1475-92.

54. Steindl P, Ferenci P, Dienes HP, Grimm G, Pabinger I, Madl C, et al. Wilson's disease in patients with liver disease: a diagnostic challenge. Gastroenterology 1997; 113: 212-8.

55. Edwards CQ, Williams DM, Cartwright GE. Hereditary hypoceruloplasminemia. Clin Genet 1979; 15: 311-6.

56. Ferenci P, Steindl-Munda P, Vogel W, Jessner W, Gschwantler M, Stauber R, et al. Diagnostic value of quantitative hepatic copper determination in patients with Wilson's disease. Clin Gastroenterol Hepatol 2005; 3: 811-8.

57. Ferenci P, Caca K, Loudianos G, Mieli Vergani G, Tanner S, Sternlieb I, et al. Diagnosis and phenotypic classiffication of Wilson disease. Liver Inter 2003; 23: 139-42.

58. Gow PJ, Smallwood RA, Angus PW, Smith AL, Wall AJ, Sewell RB. Diagnosis of Wilson's disease: an experience over three decades. Gut 2000; 46: 415-9.

59. Frommer DJ. Urinary copper excretion and hepatic copper concentrations in liver disease. Digestion 1981; 21: 169-78.

60. Martins da Costa C, Baldwin D, Portmann B, Lolin Y, Mowat AP, Mieli-Vergani G. Value of urinary copper excretion after penicillamine challenge in the diagnosis of Wilson's disease. Hepatology 1992; 15: 609-15.

61. Foruny JR, Boixeda D, López-Sanromán A, Vázquez-Sequeiros E, Villafruela M, Vázquez-Romero M, et al. Usefulness of penicillamine-stimulated urinary copper excretion in the diagnosis of adult Wilson's disease. Scand J Gastroenterol 2008; 43: 597-603.

62. Dhawan A. Evaluation of the scoring system for the diagnosis of Wilson's disease in children. Liver Int 2005; 25: $680-1$.

63. Xuan A, Bookman I, Cox DW, Heathcote J. Three atypical cases of Wilson disease: assessment of the Leipzig scoring system in making a diagnosis. J Hepatology 2007; 47: 428-33.

64. Caprai S, Loudianos G, Massei F, Gori L, Lovicu M, Maggiore G. Direct diagnosis of Wilson disease by molecular genetics. J Pediatr 2006; 148: 138-40.

65. Folhoffer A. Noninvasive diagnosis, on the basis of genetical analysis, novel mutations of ATP7B gene among 109 Hungarian patients with Wilson's disease. Eur J Gastroenterol Hepatol 2007; 19: 105-11.

66. Stapelbroek JM, Bollen CW, van Amstel JK, van Erpecum KJ, van Hattum J, van den Berg LH, et al. The H1069Q mutation in ATP7B is associated with late and neurologic presentation in Wilson disease: results of a meta-analysis. J Hepatol 2004; 41: 758-63.

67. Merle U, Schaefer M, Ferenci P, Stremmel W. Clinical presentation, diagnosis and long-term outcome of Wilson's disease: a cohort study. Gut 2007; 56: 115-20.

68. Francois D. Wilson's disease: an old disease keeps its old secrets. Eur J Gastroenterol Hepatol 2007; 19: 97-9.

69. Leggio L, Addolorato G, Abenavoli L, Gasbarrini G. Wilson's disease: clinical, genetic and pharmacological findings. Int J Immunopathol Pharmacol 2005; 18: 7-14.

70. Brewer GJ, Dick RD, Johnson V, Brunberg JA, Kluin KJ, Fink JK. Treatment of Wilson's disease with zinc: XV. Long-term follow-up studies. J Lab Clin Med 1998; 132: 264-78.

71. Ferenci P. Diagnosis and current therapy of Wilson's disease. Aliment Pharmacol Ther 2004; 19: 157-65.

72. Brewer GJ, Askari F, Lorincz MT, Carlson M, Schilsky M, Kluin KJ, et al. Treatment of Wilson disease with ammonium tetrathiomolybdate. IV. Comparison of tetrathiomolybdate and trientine in a double-blind study of treatment of the neurologic presentation of Wilson disease. Arch Neurol 2006; 63: 521-7.

73. Brewer GJ, Hedera P, Kluin KJ, Carlson M, Askari F. Treatment of Wilson disease with ammonium tetrathiomolybdate. Initial therapy in a total of 55 neurologically-affected patients and follow-up with zinc therapy. Arch Neurol 2003; 60: 379-85.

74. Medici V, Mirante VG, Fassati LR, Pompili M, Forti D, del Gaudio M, et al. Liver transplantation for Wilson's disease: the burden of neurological and psychiatric disorders. Liver Transpl 2005; 11: 1056-63.

75. Gitlin J. Wilson disease. Gastroenterology 2003; 125: 1868-77.

76. Brewer GJ, Askari FK. Wilson's disease: clinical management and therapy. J Hepatol 2005; 42 (Supl.): S13-S21.

77. Guarino M, Stracciari A, D’Alessandro R, Pazzaglia P. No neurological improvement after liver transplantation for Wilson's disease. Acta Neurol Scand 1995; 92: 405-8. 
78. Stracciari A, Tempestini A, Borghi A, Guarino M. Effect of liver transplantation on neurological manifestations in Wilson disease. Arch Neurol 2000; 57: 384-6.

79. Emiroglu R, Ayvaz I, Moray G, Karakayali H, Haberal M. Tacrolimus-related neurological complications in liver transplantation: a single center experience. Transplant Proc 2006; 38: 619-21.

80. Irani AN, Malhi H, Slehria S, Gorla GR, Volenberg I, Schilsky ML, et al. Correction of liver disease following transplantation of normal rat hepatocytes into Long-Evans Cinnamon rats modeling Wilson's disease. Mol Ther 2001; 3: 302-9.

81. Merle U, Stremmel W, Encke J. Perspectives for gene therapy of Wilson disease. Curr Gene Ther 2007; 7 : 217-20.

82. Ha-Hao D, Merle U, Hofmann C, Wesch H, Doll J, Auburger G, et al. Chances and shortcomings of adenovirus-mediated ATP7B gene transfer in Wilson disease: proof of principle demonstrated in a pilot study with LEC rats. Z Gastroenterol 2002; 40: 209-16. 